\title{
Un regard qualitatif sur la motivation en grammaire d'élèves de la fin de l'ordre élémentaire scolarisés en contexte francophone minoritaire
}

\author{
JOËL THIBEAULT \\ Université de Regina \\ CAROLE FLEURET \\ Université d'Ottawa
}

\begin{abstract}
Résumé
À partir de données provenant d'un questionnaire et d'une entrevue, cet article explore la motivation en grammaire de huit élèves de la fin de l'élémentaire en milieu minoritaire et la met en relation avec le développement de leur compétence à effectuer l'accord du verbe à l'écrit. Les résultats nous permettent de poser des hypothèses viables sur la relation entre la motivation en grammaire et le développement de ladite compétence. Ils révèlent aussi que, pour les élèves, le travail grammatical est fréquemment associé à la mémorisation de formes linguistiques et que celle-ci ne contribue pas à leur motivation.
\end{abstract}

Mots-clés

motivation, grammaire, milieu minoritaire, accord du verbe, écriture

\section{Introduction}

L'apprentissage de la langue écrite en contexte de minorité linguistique est particulier, en ce sens que le milieu socioculturel dans lequel évoluent les élèves peut influer sur le développement de la compétence qui leur permet de respecter les normes linguistiques qui sont mises en avant par l'école (Bélanger, Minor-Corriveau et Bélanger, 2015; Groupe DIEPE, 1995). Cavanagh et Blain (2009), s'attachant à mettre en exergue quelques-uns des défis que l'enseignant œuvrant en milieu linguistique minoritaire doit relever pour enseigner l'écrit, soulignent à cet égard que les apprenants (1) ont tendance à voir le français comme une langue artificielle, (2) qu'ils manquent d'occasions d'écrire en français, (3) qu'ils affichent des compétences linguistiques hétérogènes et (4) qu'ils font montre d'une certaine insécurité linguistique. Puisque, en fonction de la situation démographique de la région minoritaire et des dynamiques linguistiques qui prévalent en milieu familial, l'exposition des élèves au français à l'extérieur de l'école s'avère souvent disparate, certains chercheurs (Blain, 2003; Thibeault, 2016) soutiennent en outre que la mise en synergie des cadres théoriques offerts par la recherche en langues première (L1) et seconde (L2) serait féconde pour comprendre la réalité plurielle des apprenants qui, à la jonction d'au moins deux langues, vivent en contexte minoritaire.

Il va donc sans dire que la recherche se focalisant sur l'apprentissage de l'écriture dans ces milieux ne peut se limiter au paradigme cognitiviste qui a longtemps dominé dans cette discipline scientifique (Nelson, 2012), car l'environnement sociolinguistique et socioéducatif dans et par lequel le scripteur construit sa compétence d'écriture l'amènera à développer une relation socioaffective relativement complexe vis-à-vis du matériau écrit (Murray, 2016). Dans cet article, à la lumière de ces quelques états de fait, nous souhaitons 
nous arrêter à l'influence d'un facteur socioaffectif sur le développement de la compétence linguistique en milieu minoritaire, la motivation en grammaire. Pour ce faire, nous prendrons appui sur les résultats d'une étude de cas multiples qui a porté sur le développement de la compétence qui permet l'accord du verbe à l'écrit chez huit élèves de la fin de l'ordre élémentaire dans le sud-ouest de l'Ontario, l'une des zones les plus anglodominantes de la province (Ontario 400, 2016). Les résultats de cette étude initiale (Thibeault, Fleuret et Lefrançois, 2018; Thibeault et Lefrançois, 2018) seront dès lors utilisés dans cet article pour comprendre le rôle potentiel de la motivation en grammaire dans le développement de la compétence ciblée.

\section{La motivation, un construit complexe}

Si la recherche sur l'écriture en contexte majoritaire, en L1, a longtemps privilégié une analyse du fonctionnement cognitif par l'entremise duquel l'individu-scripteur s'engage dans le processus récursif qui le mène vers la trace graphique (Hayes et Flower, 1980), les travaux des quarante dernières années tendent à élargir leurs horizons épistémologiques et à tenir compte, notamment, de l'aspect motivationnel. De manière générale, on reconnait donc maintenant que la motivation est un élément qui contribue grandement à la production d'un texte écrit (Saddler, 2012; Troia, Shankland et Wolbers, 2012). D'après Boscolo et Hidi (2007), les nombreux construits qui relèvent de la motivation peuvent être regroupés selon trois principaux domaines, lesquels sont liés les uns aux autres et forment la dynamique motivationnelle du scripteur. Le premier a trait aux raisons qui le motivent à écrire (ses objectifs, ses intérêts, ses besoins, etc.) (Hidi, Berndorff et Ainley, 2002; Wigfield et Eccles, 1996). Le deuxième concerne le sentiment d'efficacité personnelle, cette perception que se crée le scripteur à l'endroit de sa propre compétence à écrire (Bruning, Dempsey, Kauffman, McKim et Zumbrunn, 2013; Villalón, Mateos et Cuevas, 2015). Le dernier cible les stratégies métacognitives qui lui permettent de s'autoréguler sur les plans cognitif et affectif pendant la réalisation de la tâche (p. ex., l'élaboration d'un plan, le recours à des outils de référence, la demande d'aide) (Pajares, Britner et Valiante, 2000; Zimmerman et Kitsantas, 2007).

\section{La motivation en grammaire}

Là où un nombre grandissant d'études se centrent sur l'écriture et la motivation, peu de chercheurs se penchent sur une discipline scolaire à laquelle on consacre énormément de temps en classe (Lord, 2012) et qui est souvent associée à l'écriture : la grammaire. En langue première, à notre connaissance, l'étude quantitative de Boyer (2012) est la seule qui, en français, se soit focalisée sur l'une des composantes de la motivation en grammaire et qui l'ait mise en lien avec la performance d'élèves en écriture. Plus précisément, la chercheure a examiné, chez 295 élèves de première secondaire $\left(7^{\mathrm{e}}\right.$ année) au Québec, la relation entre le biais d'évaluation de leur compétence en orthographe grammaticale et leur performance dans ce même domaine. En s'appuyant sur la recherche qui montre que l'illusion de compétence et d'incompétence tend à devenir réalité chez plusieurs élèves (Bandura, 1997; Bouffard et Narciss, 2011), Boyer a voulu voir si l'écart entre les scores des sujets lors d'une épreuve mesurant un indice d'habileté scolaire et ceux obtenus lors d'une dictée pouvait être expliqué par le sentiment d'efficacité personnelle en grammaire. Les résultats montrent que le sentiment d'efficacité est corrélé à la performance en orthographe grammaticale et que des croyances exagérément positives envers sa 
compétence orthographique sont en relation significative avec des performances supérieures en dictée. Inversement, des croyances particulièrement négatives ont eu l'effet opposé, c'est-à-dire qu'elles ont été mises en lien avec des performances inférieures en orthographe grammaticale.

En langue seconde, Jean et Simard (2011), dans une étude quantitative menée auprès de 1328 élèves de 14 ans apprenant l'anglais et de 993 élèves de 15 ans apprenant le français au Québec, ont quant à elles cherché à documenter leurs perceptions vis-à-vis de la grammaire. De manière générale, les apprenants ont une perception de l'utilité assez positive de cet objet d'apprentissage, qui serait nécessaire pour bien s'exprimer en L2, à l'oral, certes, mais encore plus à l'écrit. Cela dit, l'intérêt qui est manifesté par les participants en ce qui a trait à la grammaire demeure restreint, ce qui pousse les chercheures à soutenir que, pour les élèves, l'enseignement grammatical demeurerait un mal nécessaire : ils en perçoivent l'utilité, bien qu'il soit une source d'intérêt restreinte.

À notre connaissance, aucune recherche adoptant un devis qualitatif n'a tenté de décrire la dynamique motivationnelle des élèves en grammaire. De telles études, cela étant, pourraient nous informer en ce qui a trait aux pratiques qui sont les plus susceptibles de motiver les apprenants et qui, de facto, répondraient à leurs besoins socioaffectifs. En milieu minoritaire, par ailleurs, on sait que les élèves font souvent face à une insécurité linguistique importante (Cavanagh et Blain, 2009) et, pour cette raison, il nous semble important que la recherche s'intéresse à la motivation des élèves en grammaire. Ainsi, dans le présent article, nous visons à rendre compte de la motivation en grammaire chez des élèves scolarisés en français là où cette langue est minoritaire et à observer s'il existe une relation entre cette motivation et le développement de la compétence linguistique qui permet l'accord du verbe à l'écrit.

\section{Une note sur l'accord du verbe à l'écrit}

De prime abord, le choix de cet objet linguistique peut sembler anodin. Après tout, la règle qui en régit le fonctionnement - le verbe s'accorde en personne et en nombre avec le noyau du groupe en fonction sujet (Chartrand, Aubin, Blain et Simard, 2011) - parait relativement simple. Or, la recherche montre que son apprentissage est chronophage et qu'il n'est pas terminé à la fin de l'élémentaire (Cogis, 2013; Geoffre et Brissaud, 2012), quoique les instances ministérielles ontariennes en demandent la maitrise à ce moment de la scolarité obligatoire (ministère de l'Éducation de l'Ontario, 2006). Afin d'expliquer cette complexité, David et Renvoisé (2010) nous rappellent notamment que les verbes au présent de l'indicatif affichent de nombreuses marques inaudibles et qu'un même verbe peut détenir plusieurs radicaux (p. ex., le verbe aller en compte quatre au présent de l'indicatif). Il nous semblait donc intéressant de nous arrêter à l'accord du verbe puisque, au terme de l'école élémentaire, les élèves doivent avoir construit des connaissances à son endroit, mais que, pour plusieurs, cet apprentissage est encore en cours à ce moment. On peut donc s'interroger sur l'influence de facteurs comme la motivation en grammaire, lesquels peuvent soutenir ou freiner l'appréhension de cet objet linguistique complexe.

\section{Méthodologie}

Comme nous le disions en introduction, l'étude dont il est question ici repose sur une recherche plus large, dont l'objectif était de décrire le développement de la compétence permettant l'accord verbal à l'écrit chez des élèves de la fin de l'élémentaire en contexte 
francophone minoritaire (Thibeault, Fleuret et Lefrançois, 2018; Thibeault et Lefrançois, 2018)

Nos participants, quatre filles et quatre garçons, étaient tous scolarisés dans une classe à niveaux multiples du sud-ouest de l'Ontario au moment de la recherche, durant l'année scolaire 2015-2016. Pour composer notre échantillon, nous avons abordé le conseil scolaire, qui nous a référé à l'enseignante des participants. Nous avons ensuite invité l'ensemble des élèves de la classe à prendre part à l'étude; nous avons reçu l'autorisation parentale de ces huit élèves. Au commencement de la recherche, la moitié d'entre eux était en cinquième année, l'autre était en sixième année, et nous les avons suivis pendant 13 mois pour décrire le développement de la compétence par le truchement de laquelle ils opéraient, à l'écrit, l'accord du verbe au présent de l'indicatif. Ils ont tous une intelligence normale en regard des épreuves du K-ABC (Kaufman et Kaufman, 1995), auxquelles ils ont été soumis pendant la recherche. Tous sont plurilingues, connaissant minimalement le français et l'anglais. Six ont toujours été scolarisés en français en Ontario, alors qu'Isaac est arrivé de la République d'Ouganda, où il a appris le français langue seconde avant son arrivée au Canada en cinquième année, et que Pierre a suivi un programme de scolarisation bilingue français-créole haïtien avant d'arriver au Canada en $4^{\mathrm{e}}$ année. Dans le tableau 1 sont répertoriées les langues qu'utilisent les élèves au foyer avec leurs parents. Notons que, afin de préserver leur anonymat, nous avons remplacé le nom des participants par des pseudonymes.

Tableau 1

Langue(s) que les participants utilisent lorsqu'ils parlent à leurs parents

\begin{tabular}{|c|c|c|}
\hline $\begin{array}{l}\text { Élève } \\
\text { (niveau scolaire } \\
\text { en début d'étude) }\end{array}$ & $\begin{array}{l}\text { Langue(s) parlée(s) } \\
\text { avec la mère }\end{array}$ & $\begin{array}{l}\text { Langue(s) parlée(s) } \\
\text { avec le père }\end{array}$ \\
\hline $\operatorname{Emma}\left(5^{\mathrm{e}}\right)$ & Anglais & Anglais \\
\hline Maya $\left(5^{\mathrm{e}}\right)$ & Anglais, français & Anglais \\
\hline $\operatorname{Ali}\left(5^{\mathrm{e}}\right)$ & Arabe, français, anglais & Anglais, arabe, français \\
\hline Alicia $\left(5^{\mathrm{e}}\right)$ & Anglais, tagalog, français & Anglais \\
\hline Sabrina $\left(6^{\mathrm{e}}\right)$ & Anglais, français, espagnol & Anglais \\
\hline Pierre $\left(6^{\mathrm{e}}\right)$ & Créole haïtien & Créole haïtien \\
\hline Kate $\left(6^{\mathrm{e}}\right)$ & Anglais, français & Anglais \\
\hline Isaac $\left(6^{e}\right)$ & Swahili, français & Français, anglais \\
\hline
\end{tabular}

Pour explorer leur motivation en grammaire, nous avons recouru à deux instruments, ces derniers ayant étant élaborés en fonction de trois axes : l'intérêt de l'élève vis-à-vis de la grammaire, sa perception de l'utilité de la grammaire et son sentiment d'efficacité en grammaire. Le contenu de ces deux outils a fait l'objet d'une validation, une didacticienne de la grammaire et une psychologue s'intéressant à la motivation ayant gracieusement accepté d'y jeter un regard critique. Nous les avons aussi mis à l'essai dans le cadre d'une étude-pilote menée auprès de sept élèves de $5^{\mathrm{e}}$ année d'une autre classe de la même école. À la suite de la validation de contenu et de la pilote, nous avons apporté les 
quelques modifications que nous jugions nécessaires et, en mai 2015, nous avons invité nos huit participants à prendre part à une collecte ciblant leur motivation en grammaire.

Le premier instrument que nous avons conçu est un questionnaire à questions fermées de type Harter (1999) dans le cadre duquel le participant doit lire un énoncé, faire un choix binaire et, ensuite, nuancer son positionnement initial. Dans un premier temps, il doit donc choisir, selon l'énoncé, dans quel groupe il se situe, celui des cercles ou des carrés. Puis, il se positionne à nouveau, selon son degré d'appartenance au groupe des cercles ou des carrés. La figure 1 présente un exemple d'item portant sur la perception de l'utilité de la grammaire.

Dans le groupe des cercles, les élèves pensent que la grammaire n'est pas utile.

Dans le groupe des carrés, les élèves pensent que la grammaire est utile.

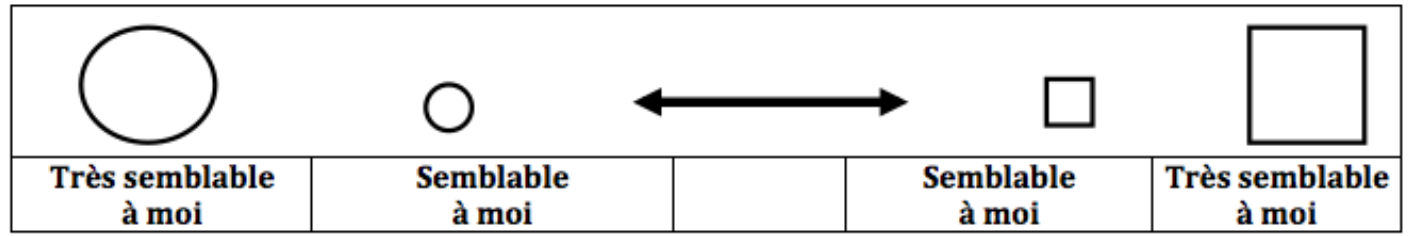

Figure 1. Exemple d'item du questionnaire sur la motivation en grammaire

Pour ce qui est du contenu du questionnaire, on retrouve quatre items pour chacun des trois axes que nous avons retenus pour aborder la motivation en grammaire ; ainsi compte-t-il un total de 12 items. Chacun d'eux a été codé à l'aide d'une échelle de -2 à +2 , le total possible s'échelonnant donc de -24 à +24 , de -8 à +8 par axe.

Pour compléter les données en lien avec la motivation en grammaire, et pour avoir un accès privilégié au cadre personnel de référence de nos participants quant à ce construit (Van der Maren, 2004), nous les avons également rencontrés individuellement à la bibliothèque de leur école dans le cadre d'un entretien semi-dirigé. Gravitant autour des trois axes que nous avons arrêtés précédemment, cet entretien nous offrait l'occasion, entre autres, de colliger des données témoignant de leur expérience et de leurs points de vue (Baribeau et Royer, 2012). Le guide d'entretien est constitué de cinq questions et, selon les réponses que nous proposaient les participants pendant l'entrevue, nous leur posions des relances afin d'obtenir davantage d'informations relativement au construit que nous abordions avec eux. Deux questions traitaient de l'intérêt en grammaire («Qu'est-ce que tu aimes faire en grammaire à l'école ? Qu'est-ce que tu n'aimes pas faire en grammaire à l'école ? »), une de la perception de l'utilité de la grammaire («D'après toi, la grammaire, ça sert à quoi ? »), deux de la perception de sa compétence en grammaire («Qu'est-ce qui est difficile pour toi en grammaire? Qu'est-ce qui est facile pour toi en grammaire ?»).

Comme nous l'avons mentionné précédemment, cette étude s'inscrit dans une recherche plus large (Thibeault, Fleuret et Lefrançois, 2018; Thibeault et Lefrançois, 2018), lors de laquelle nous avons décrit le développement de la compétence permettant 
l'accord du verbe à l'écrit de nos participants ${ }^{1}$. Ainsi cette étude initiale nous a-t-elle permis de classer nos huit participants en trois groupes de scripteurs distincts. Le premier, constitué d'Emma, de Pierre et de Sabrina, affiche une compétence opérationnelle dès le début de la recherche et s'engage fréquemment dans la quête du donneur d'accord pour opérer l'accord du verbe; la compétence de ces élèves n'évolue que légèrement au gré des 13 mois de la recherche. Plus faible, le deuxième groupe, celui de Kate, de Maya et d'Isaac, en début de recherche, ne connait pas la marque prototypique de la pluralité des verbes (ent) et ne s'engage pratiquement jamais dans la quête d'un potentiel donneur pour les accorder ; ils utilisent surtout la chaine parlée ( Je l'ai écrit comme ça se prononce») et le temps verbal ( « Je l'ai écrit comme ça parce que c'est le présent ») afin de justifier leurs graphies. À la fin de la recherche, on note chez ces scripteurs une intériorisation progressive du morphème de la pluralité des verbes, mais son recours est encore loin d'être stabilisé, et la réflexion grammaticale dont ils font preuve n'est pas centrée sur l'identification du donneur d'accord. In fine, le troisième groupe, Ali et Alicia, ressemble au deuxième au commencement de l'étude. Cela dit, entre mai 2015 et février 2016, il accuse un développement notable et, en fin de recherche, il rappelle davantage le premier groupe. Pour ce texte, sans présenter dans le détail les données décrivant le développement de la compétence associée à l'accord verbal, nous prendrons appui sur les regroupements de scripteurs qui ont émergés lors de l'étude initiale et nous focaliserons notre attention sur les résultats liés à la motivation en grammaire.

\section{Résultats}

Pour rendre compte de nos données, nous nous arrêterons d'abord aux résultats globaux des élèves au questionnaire. Par la suite, nous nous concentrerons sur chacun des trois indicateurs de la motivation en grammaire et nous présenterons le score des élèves pour chacun d'eux. La présentation des résultats relatifs aux indicateurs sera également accompagnée d'extraits de verbatim tirés des entretiens individuels, ces passages complétant et nuançant donc les données provenant du questionnaire.

\footnotetext{
${ }^{1}$ Pour décrire ce développement, nous avons recouru à trois outils : l'activité de complètement, lors de laquelle l'élève devait écrire des verbes au présent de l'indicatif dont l'infinitif apparait entre parenthèses, une production écrite et un entretien métagraphique, dans le cadre duquel nous avons demandé aux participants de verbaliser les raisonnements qu'ils ont mis en œuvre pour accorder les verbes lors des deux tâches précédentes. Chaque instrument a été utilisé à trois reprises, sur une période de 13 mois. Qui plus est, nous avons demandé à l'enseignante des élèves de procéder à son enseignement habituel de l'accord du verbe.
} 


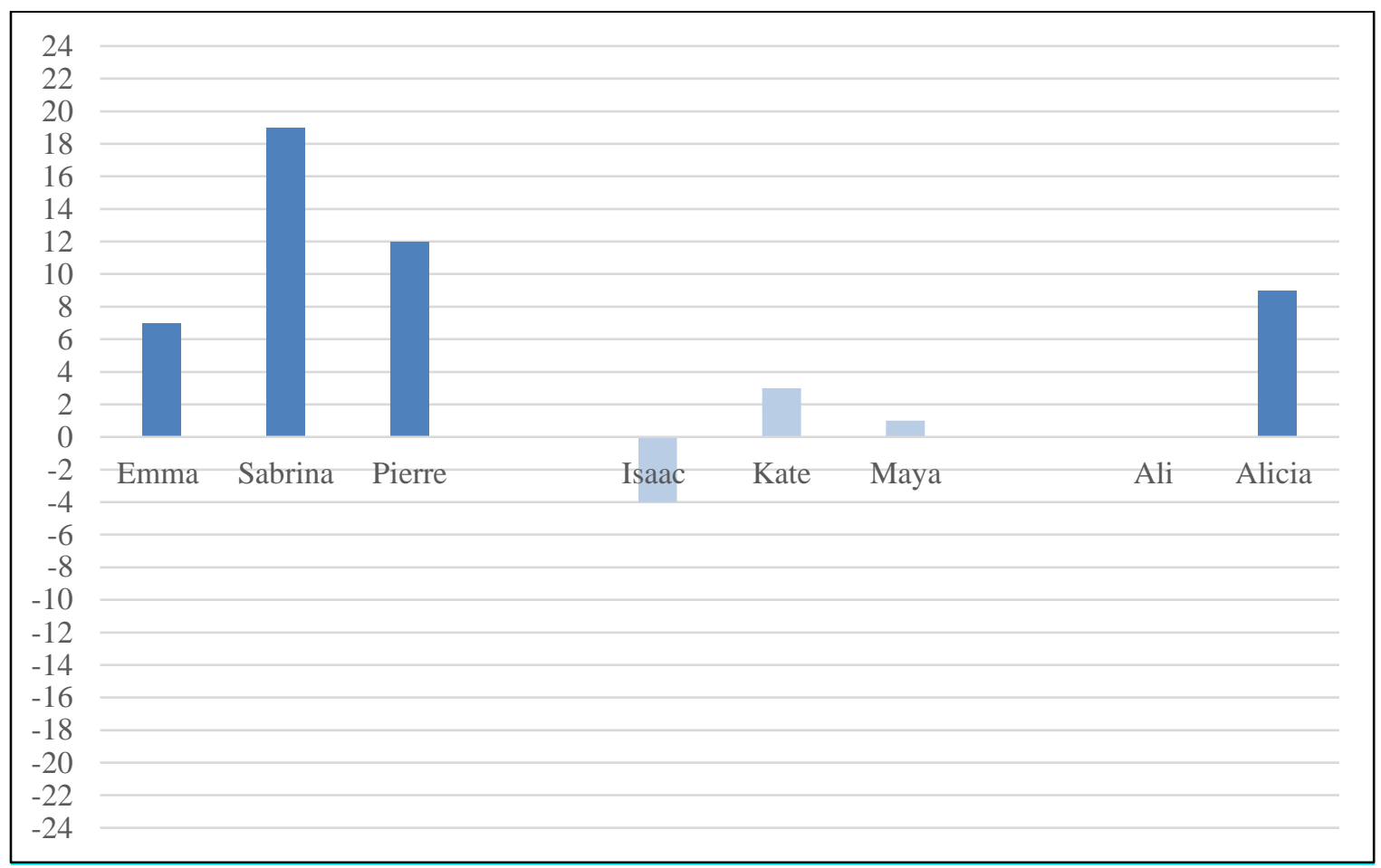

Figure 2. Scores globaux de la motivation en grammaire

Les élèves du groupe 1, celui qui fait montre d'une compétence assurée tout au long de l'étude, témoignent d'une motivation claire en grammaire, leur score se situant entre $7 / 24$, pour Emma, et 19/24, pour Sabrina. Pour ce qui est des élèves du deuxième regroupement, ceux dont la compétence ciblée est peu opérationnelle au gré de la recherche, les résultats sont plus faibles et homogènes, se situant entre -4/24, pour Isaac, et $3 / 24$, pour Kate. La motivation des participants du troisième groupe diffère quant à elle d'un élève à l'autre; Ali obtient un score de 0/24, tandis qu'Alicia obtient 9/24. Il est donc difficile de proposer des explications relatives à la dynamique motivationnelle des élèves $\mathrm{du}$ troisième groupe en grammaire et aux potentiels liens qu'elle entretient avec leur compétence à accorder les verbes. Cela dit, le portrait global que nous brossons permet de poser des hypothèses concernant les relations entre la motivation en grammaire et la compétence permettant l'accord verbal. En effet, tous les élèves du groupe 1 semblent être relativement motivés dans leur apprentissage des composantes grammaticales de la langue, alors que les élèves du groupe 2 le sont moins. Les élèves du groupe 3, de leur côté, présentent des résultats qui fluctuent.

\section{L'intérêt en grammaire}

Premier indicateur de la motivation en grammaire, l'intérêt des élèves à l'égard de cette discipline parait quelque peu mitigé, comme on le constate à partir de la figure 3. Sabrina (7/8), Maya (7/8) et Pierre (5/8) présentent des scores élevés, les autres en affichent des plus faibles. Alicia (2/8) et Kate (2/8) obtiennent des scores positifs, mais moindres, alors que ceux des autres participants, Emma (-1/8), Ali (-4/8) et Isaac (-5/8), sont négatifs. 


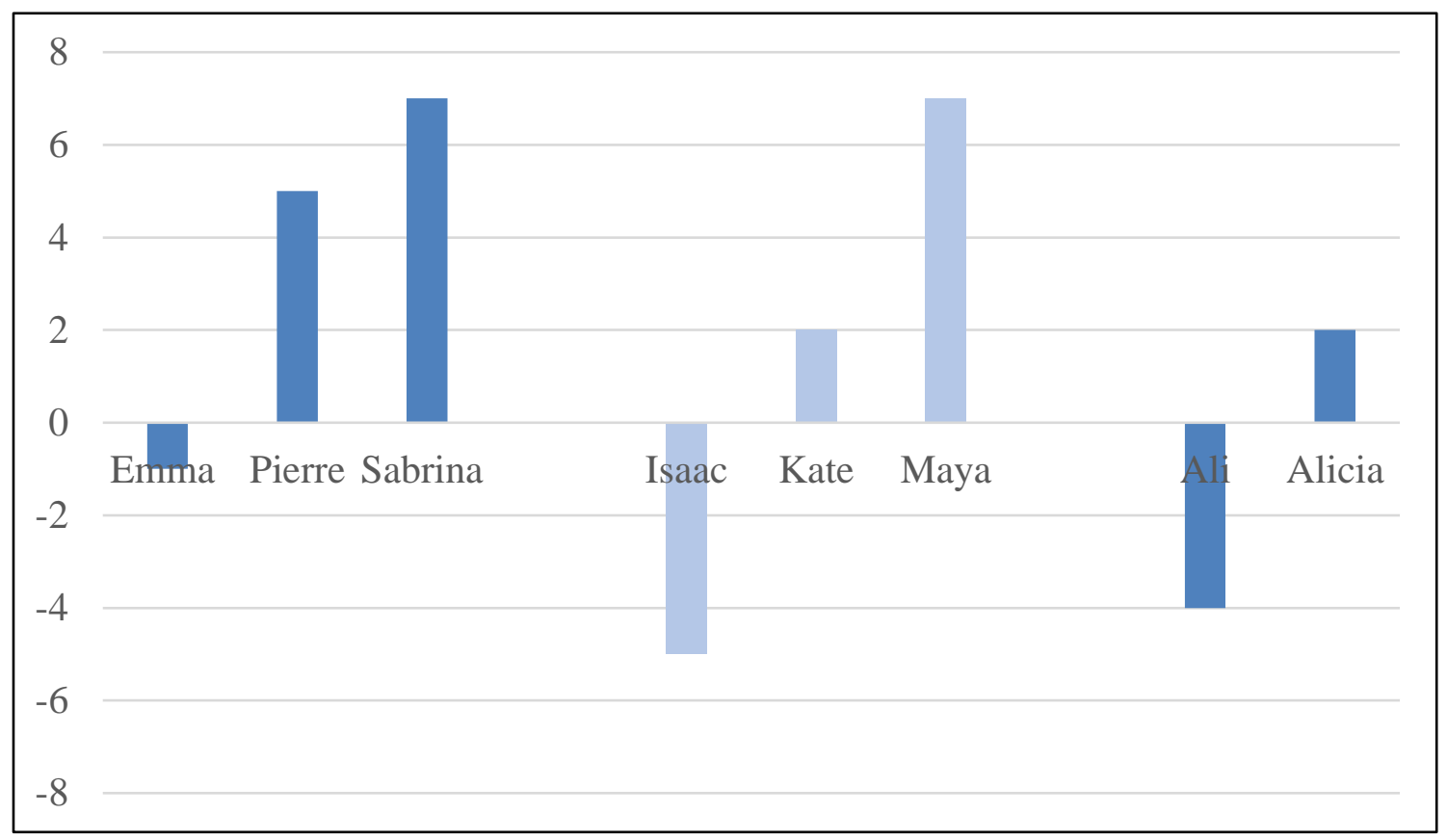

Figure 3. Scores liés à l'intérêt en grammaire

Dans le groupe 1, on retrouve une disparité importante concernant l'intérêt en grammaire. Emma, qui obtient le score le plus faible du regroupement, souligne qu'elle aime apprendre les adjectifs.

Expérimentateur : Qu'est-ce que tu aimes faire en grammaire à l'école ?

Emma : J'aime faire les adjectifs.

Expérimentateur : Les adjectifs. Pourquoi les adjectifs ?

Emma: Parce que comme, il y a beaucoup de différents types d'adjectifs, so c'est le fun d'apprendre.

Expérimentateur : Qu'est-ce qui est le fun d'apprendre dans les adjectifs ?

Emma: Ils sont faciles. Comme, ça décrit un chose, une personne. Il y a beaucoup de différentes sortes, et c'est le fun d'apprendre les différents et qu'est-ce qu'ils décrivent.

Dans ce passage, on note que c'est le travail lexical qui semble être d'intérêt pour Emma. En effet, quoiqu'elle fasse mention des adjectifs, elle n'en discute pas en termes de morphosyntaxe, mais en fonction du sens qu'ils véhiculent et des informations qu'ils apportent aux noms qu'ils complètent. Quand nous l'interrogeons sur ce qu'elle n'aime pas en grammaire, elle précise que les autres notions grammaticales lui déplaisent parce qu'elle doit constamment s'investir dans un effort de mémorisation lorsqu'elle les apprend.

Pierre et Sabrina, dont les scores sont relativement similaires pour ce qui est de l'intérêt en grammaire, présentent un discours différent de celui d'Emma ; on remarque en effet qu'ils prennent plaisir à s'engager dans une réflexion grammaticale et qu'ils aiment comprendre le fonctionnement d'unités linguistiques. 
Expérimentateur : Qu'est-ce que t'aimes faire quand tu fais le complément de phrase ?

Pierre: Tracer les lignes.

Expérimentateur: Tracer les lignes ?

Pierre: Pour que ça montre où ça va. C'est l'fun.

Expérimentateur: Pourquoi tu trouves ça le fun?

Pierre : Tu peux écrire si c'est bon ou non. Tu peux même dire si je dois mettre un virgule.

Dans cet extrait, Pierre fait référence à l'analyse de phrases et aux lignes qu'il doit tracer afin de rendre compte de sa démarche. Il montre à cet égard qu'il connait le fonctionnement syntaxique du complément de phrase et explique que l'analyse de ce dernier, qu'il réalise en traçant des lignes, est pour lui une source d'intérêt.

Pour ce qui est du groupe 2, dans lequel on note aussi une variation considérable dans les scores liés à l'intérêt en grammaire, la réflexion métalinguistique n'apparait pas dans le discours des élèves. Isaac, qui obtient le score le plus bas du regroupement, souligne qu'il prend plaisir à apprendre les verbes :

Expérimentateur: Pourquoi tu aimes conjuguer les verbes?

Isaac: Conjuguer les verbes, c'est amusant, ça t'aide aussi à savoir comment...comme si tu conjugues ce verbe au présent comme...comment est-ce que...qu'est-ce qui change.

Expérimentateur : Okay, qu'est-ce qui change où ?

Isaac: Comme si c'est un partie ou c'est tout le mot qui change.

Comme Emma, Isaac souligne qu'il aime se concentrer sur une catégorie de mot, ici le verbe ; en effet, il appert que la modification du verbe en fonction du contexte phrastique suscite chez lui un intérêt marqué. Quand nous lui demandons ce qu'il lui déplait, il nomme la correction de ses productions.

Isaac : Je n'aime pas comme...je n'aime pas écrire et corriger les mots si comme tu te trompes. Si tu dois conjuguer, tu dois le changer dans le texte, c'est comme conjuguer, mais c'est corriger en grammaire.

Expérimentateur : Corriger en grammaire, et pourquoi tu n'aimes pas ça ?

Isaac: Car moi je pense que si je pouvais savoir comment comme...si je pouvais retenir tout le grammaire avant de faire mon test, ça m'aidera à me complètement corriger.

Isaac semble donc associer le développement de connaissances déclaratives, la mémorisation des règles grammaticales, aux résultats qu'il obtient dans le cadre d'examens et aux corrections qu'il effectue de ses productions. Or, cette mémorisation semble lui poser problème et affecter son intérêt pour la grammaire.

Eu égard au groupe 3, qui accuse également une certaine disparité dans les scores liés à l'intérêt, les réponses des élèves pendant l'entrevue varient. Ali, qui obtient un score bas au questionnaire, mentionne qu'il apprécie écrire et analyser des phrases, parce que c'est plaisant pour lui. Quand on le questionne concernant ce qui lui déplait, il discute du soutien que lui offre son enseignante. 
Expérimentateur : Qu'est-ce que t'aimes pas faire en grammaire à l'école ?

Ali : Euh, comme, quand, tu sais quand on analyse les phrases, des fois, well, on doit le faire tout seul. Je demande de l'aide pour comme, une vraiment longue phrase qui a comme deux verbes et comme trois sujets ou quelque chose...like...like...sometimes, I ask her to help me, and it takes forever.

Expérimentateur: Okay, donc le faire seul, you don't really like it?

Ali : Non, j'aime faire seul, mais quand je demande de l'aide, ça prend comme... ça me slow down.

Il semble donc qu'Ali aime s'engager dans une réflexion métalinguistique, car il mentionne l'analyse de phrases comme étant un vecteur d'intérêt en grammaire. Cela étant, quand il fait face à des difficultés, il aimerait recevoir un appui promptement.

\section{La perception de l'utilité de la grammaire}

Eu égard de la perception de l'utilité de la grammaire, les scores sont globalement plus élevés que ceux associés à l'intérêt. La figure 4 en fait justement état. Cette fois-ci, Maya obtient le score maximal (8/8); elle est suivie de Sabrina (7/8), et d'Emma, de Pierre et d'Alicia, qui ont tous le même score, 6/8. Viennent ensuite Kate (5/8), Isaac (2/8) et Ali (-4/8), ce dernier participant étant le seul qui, pour cet indicateur de la dynamique motivationnelle en grammaire, présente un score négatif.

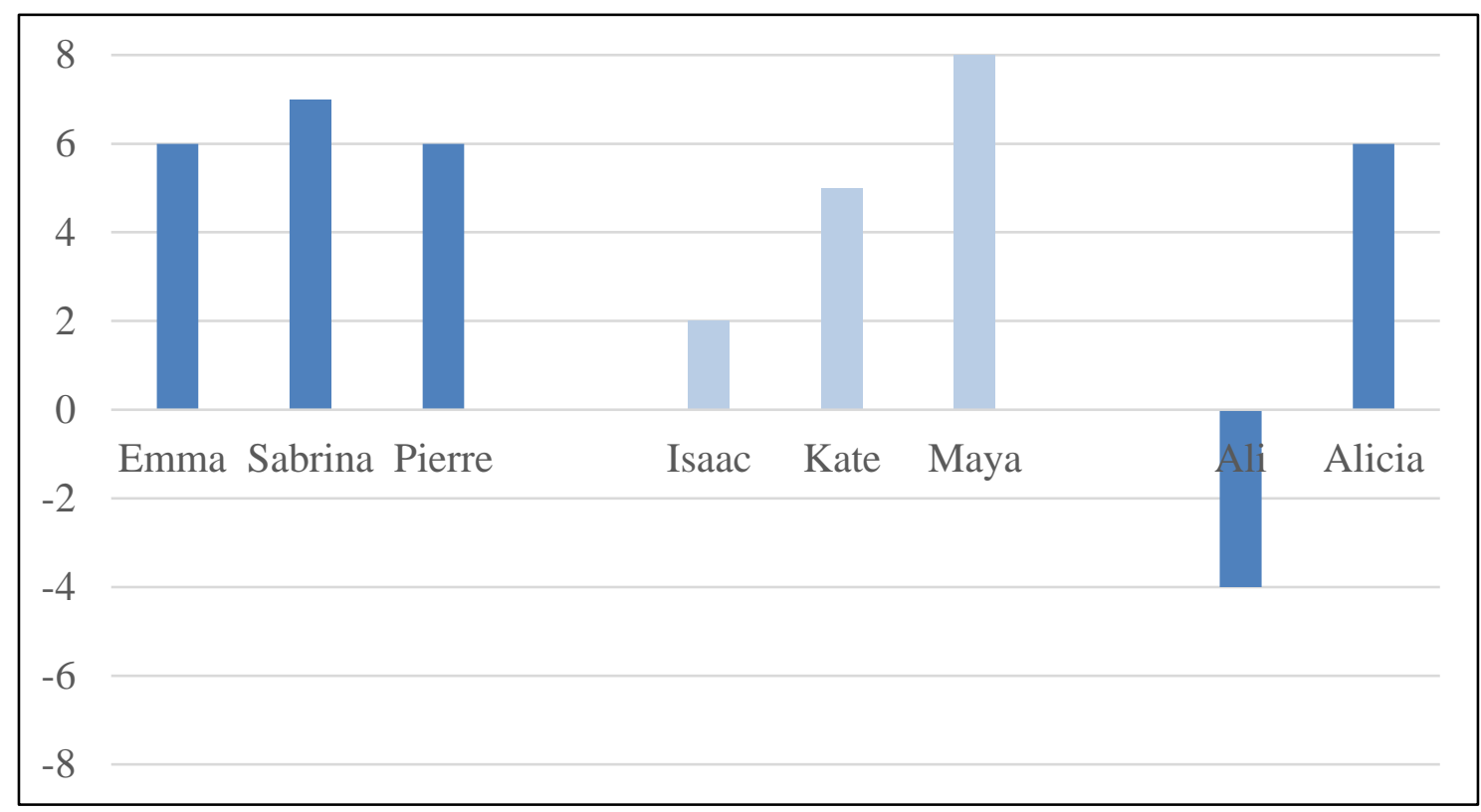

Figure 4. Scores liés à la perception de l'utilité de la grammaire

Les trois élèves du premier groupe 1 semblent avoir développé une perception positive face à l'utilité de la grammaire. Tous ces participants affirment que l'enseignement grammatical leur permet de parfaire la qualité de leurs productions. Sabrina explique :

Expérimentateur : Alors, dis-moi Sabrina, la grammaire, pour toi, ça sert à quoi ? 
Sabrina: Euh...ça sert pour, comme, quand je veux parler, je peux utiliser comme le...euh les bons mots et comme, si je voulais dire, comme la chaise, je pouvais aussi en train de dire le chaise.

Dans ce passage, non seulement Sabrina nomme-t-elle une utilité de la grammaire, celle en lien avec la bonification de ses discours oraux, elle cible aussi un fait de langue, l'accord du déterminant en genre, qu'elle utilise en exemple ${ }^{2}$. En fait, à la suite de ce court extrait, elle discute également de la conjugaison et de la position de l'adjectif dans le groupe nominal.

Le questionnaire nous dévoile que les différents élèves du groupe 2 se sont construit des perceptions différentes de la grammaire. Contrairement aux élèves du groupe 1, ils ne font jamais explicitement mention de faits de langue. Isaac, par exemple, souligne que la grammaire lui est utile afin de comprendre les mots et, plus généralement, de connaitre la langue.

Expérimentateur : Est-ce que ça sert à autre chose ?

Isaac: Tu peux savoir leur langue. Tu vois comment écrire dans des différentes langues.

Il nous est difficile de faire sens de ces propos d'Isaac, qui a obtenu le score le plus faible de son regroupement. Il est tout de même intéressant qu'il ne se limite pas, dans son discours, à l'apprentissage du français, la grammaire lui étant principalement enseignée dans cette langue. Peut-être veut-il dire que l'apprentissage grammatical contribue au développement de compétences rédactionnelles dans plusieurs langues, mais, sans précisions de sa part, nous ne pouvons pas nous avancer de manière définitive.

À l'instar d'Isaac, Kate, qui obtient trois points de plus que son camarade au questionnaire, souligne que la grammaire lui permet d'écrire des textes; elle ne fait toutefois pas mention d'autres langues que le français.

Expérimentateur: Ma première petite question... d'après toi Kate, faire de la grammaire en classe là, ça sert à quoi?

Kate : Pour que, quand on est plus vieux que comme, si on écrit des textes, si on peut comme écrire les histoires.

Bien qu'elle reconnaisse cette utilité scripturale, il appert qu'elle l'associe à la vie adulte, qu'elle n'en perçoit pas l'utilité immédiate. Cet état de fait trouve un écho certain dans la suite de l'entrevue, quand nous lui demandons de nous faire part d'autres utilités de la grammaire.

Expérimentateur : Est-ce que ça sert à autre chose?

Kate : Euh...like, for jobs.

${ }^{2}$ Cet exemple est intéressant, car il présente une difficulté qui est principalement attestée chez des locuteurs du français langue seconde. En effet, le choix du genre du déterminant, qui relève de l'assignation du genre des noms, n'est que peu documenté en L1, puisque les élèves ont souvent construit les connaissances implicites permettant la sélection du genre nominal. Cela, à notre avis, montre la pertinence d'un arrimage conceptuel L1/L2 pour les milieux francophones minoritaires (Blain, 2003; Thibeault, 2016). 
Expérimentateur : For jobs? Okay. Et autre chose? How is it useful for jobs ?

Kate : Comme, si tu dois comme, enseigner le grammaire, de le savoir. Si tu travailles n'importe où... you have to take notes and give them to people. You have to know, like, how to write.

On remarque encore une fois dans cet extrait que la grammaire, pour Kate, est utile, certes, mais qu'elle en voit principalement une praticité, celle de pouvoir remplir les tâches qui sous-tendent un potentiel emploi.

Au questionnaire, les membres du groupe 3, Ali et Alicia, affichent des scores distincts, cette dernière percevant davantage les bénéfices de la grammaire. Ali semble tout de même en reconnaitre deux utilités, la première linguistique, l'autre scolaire. Il postule d'entrée de jeu que cet objet d'apprentissage lui vient en aide lorsqu'il écrit et lit des textes. Concernant l'écriture, nous avons recueilli les propos suivants.

Expérimentateur : Comment ça t'aide à écrire des histoires ?

Ali : Euh, parce que, j'aime écrire beaucoup, et dans des tests, tests d'écriture, yeah, je dois savoir comment... les... analyser les phrases. Et tests de grammaire, je dois savoir quoi ce que, comment on utilise les phrases.

Il mentionne donc explicitement un intérêt pour l'écriture, mais il met l'accent sur l'utilité de la grammaire en fonction des examens qu'il doit compléter à l'école, notamment en syntaxe. Concernant la lecture, il précise ceci :

Expérimentateur : Puis comment, tu as dit que quand tu lis aussi, ça t'aide. Comment ça t'aide quand tu lis?

Ali : Parce que je peux savoir comment les prononcer.

Expérimentateur : Est-ce que tu peux m'en dire plus ?

Ali : Euh, parce que comme, euh, well, maintenant on faisait le mot écureuil. Je lisais le phrase et j'étais comme, je ne savais pas comment le prononcer parce qu'il y avait un $e$ à la fin et j'étais comme, l'écureul ou écureuil ?

Expérimentateur : Et, donc, la grammaire t'aide à pouvoir le prononcer correctement ?

Ali : Oui.

Dans ce passage, Ali déclare que la grammaire lui vient en aide dans l'association des graphèmes aux phonèmes correspondants ; on peut dès lors questionner ce qu'est la grammaire pour cet élève, sa définition allant au-delà du domaine linguistique qui y est traditionnellement associé, la morphosyntaxe.

Pour terminer, soulignons qu'Alicia, qui obtient un score d'utilité plus élevé qu'Ali, reconnait les apports de la grammaire dans l'élaboration de discours dotés de sens.

Expérimentateur : Alicia, pour toi, dis-moi, faire de la grammaire en classe, ça sert à quoi? 
Alicia : Ça sert comme, quand tu dis le, quand tu dis le sentence, ça fait plus de sens...

Expérimentateur : Okay.

Alicia : ...et tu peux comme understand qu'est-ce qu'on va dire, comme ça, ya.

On remarque donc que, contrairement à Ali, ce n'est pas l'association graphophonémique qui lui vient à l'esprit quand nous la questionnons sur l'utilité de la grammaire, c'est plutôt la construction de sens que cet objet facilite pour elle.

\section{Le sentiment d'efficacité personnelle en grammaire}

Les résultats de nos huit participants quant à leur sentiment d'efficacité personnelle en grammaire sont globalement plus bas que ceux associés à leur perception de son utilité. Le figure 5 présente les scores qu'ils ont obtenus.

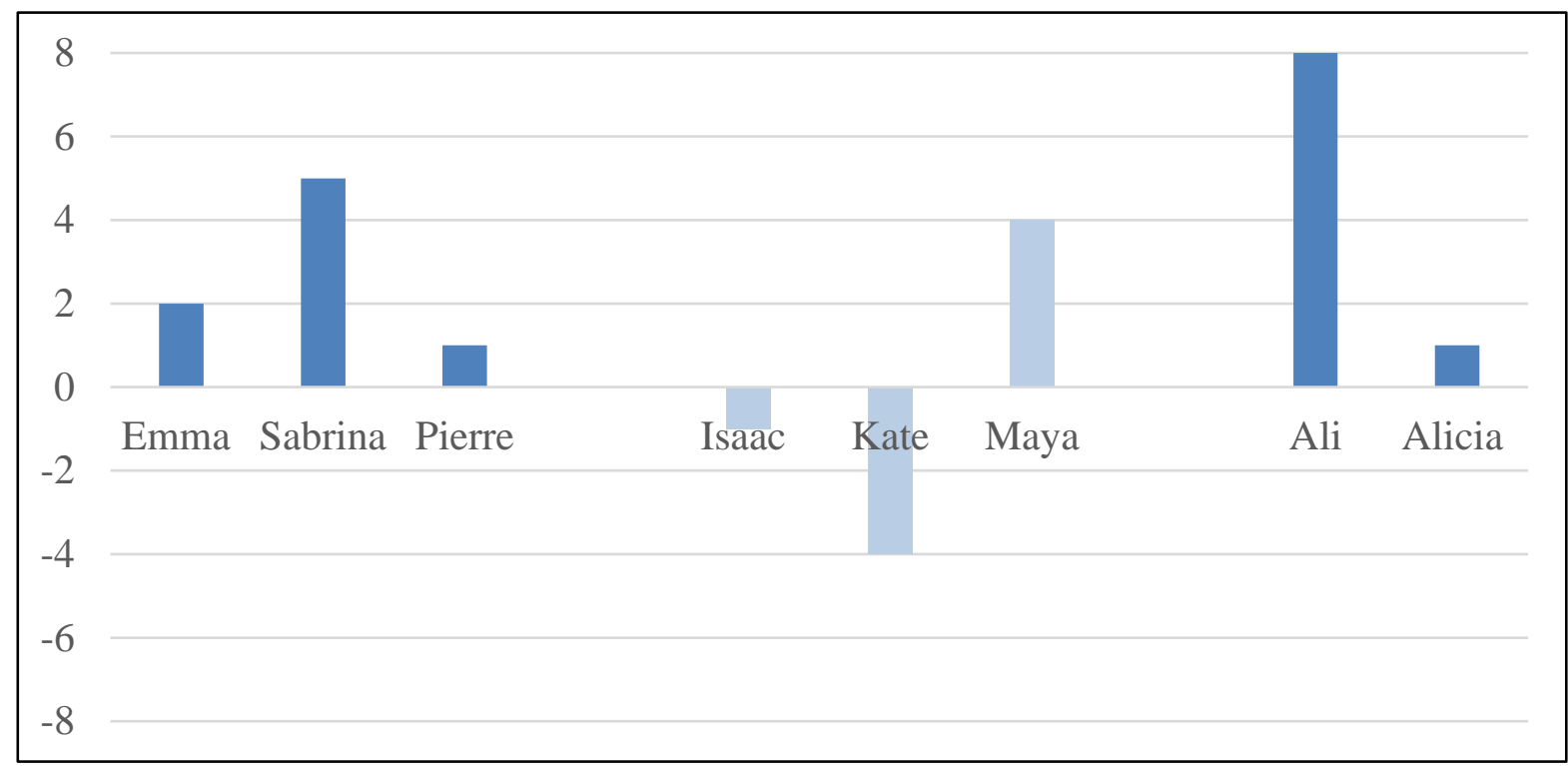

Figure 5. Scores liés au sentiment d'efficacité personnelle en grammaire

Cette fois-ci, alors qu'il était parmi les élèves dont les scores étaient les plus bas pour ce qui est des indicateurs précédents, Ali obtient le score le plus élevé, $8 / 8$. Il est suivi de Sabrina (5/8) et de Maya (4/8), qui sont encore une fois parmi les scripteurs dont le résultat est le plus haut. Viennent ensuite Emma (2/8), Pierre et Alicia (1/8), Isaac (-1/8) et Kate (-4/8).

Disparates, les scores des élèves du groupe 1 sont tous positifs. Pour Emma, qui nous affirme être compétente en grammaire, c'est l'étude des verbes qui est facile, bien qu'elle ne sache pas nous dire pourquoi. Du côté de Sabrina, celle qui obtient le score le plus élevé du groupe au questionnaire, l'analyse de phrases semble revêtir une certaine facilité.

Expérimentateur: Qu'est-ce qui est facile pour toi en grammaire? 
Sabrina: Facile? Facile c'est, c'est comme, essayer de trouver le sujet, le verbe et le prédicat et des choses dans les phrases.

Expérimentateur : Okay. Pourquoi c'est facile, ça?

Sabrina: C'est facile car euh, car on utilise comme des petites machines. Alors c'est plus, alors c'est plus facile à savoir, comme, et tu dois te poser des questions, comme, pour savoir si c'est un sujet ou le verbe ou le prédicat.

Les machines dont parle Sabrina renvoient à un outil référentiel que son enseignante utilise pour aider ses élèves à poser des hypothèses sur les fonctions grammaticales et les catégories de mots, et à les valider (Lefrançois, Montésinos-Gelet et Anctil, 2018). Dans cette machine, par exemple la machine à sujet, se trouvent les questions que l'élève doit se poser pour vérifier si un groupe de mots est bel et bien le sujet de la phrase (Puis-je l'effacer? Puis-je l'encadrer par c'est...qui ?, etc.). Il appert donc que cet outil, qui repose sur la réflexion métalinguistique de l'élève, facilite l'apprentissage grammatical de Sabrina. Par ailleurs, quand on la questionne concernant ses difficultés en grammaire, elle mentionne le choix des radicaux des verbes et la mémorisation qui lui est associée.

Pierre, qui affiche le sentiment d'efficacité personnelle le plus faible au questionnaire, tient des propos fort différents de ceux de ses pairs du groupe 1. Contrairement à elles, il affirme que les verbes sont faciles pour lui.

Expérimentateur : Qu'est-ce qui est facile pour toi en grammaire ?

Pierre: Verbes.

Expérimentateur: Et pourquoi cela?

Pierre : Tu dois les savoir, tu dois les savoir par cœur.

Il avance aussi que le repérage du verbe est facile, car il peut prendre appui sur la terminaison du mot, qui lui en indique la catégorie grammaticale. Pour ce qui est de ses difficultés, il fait mention de l'identification du prédicat de phrase.

Expérimentateur: Pourquoi tu ne peux pas le trouver (le prédicat)?

Pierre : Non, comme, le verbe si tu le trouves, tu peux trouver le prédicat facile, mais si tu ne peux pas trouver le verbe, tu ne peux pas trouver le prédicat de phrase.

Le prédicat, fonction de premier niveau et constituant de la phrase syntaxique française, ne peut être repéré à l'aide de manipulations décisives, comme c'est le cas, par exemple, du sujet. Quand la phrase est de type déclaratif, l'élève peut donc procéder par élimination, en identifiant le sujet et les potentiels compléments de phrase, et en décrétant que le groupe restant de cette analyse est le prédicat. Il peut aussi, comme le précise Pierre, repérer le verbe, ses compléments et ses modificateurs, et postuler qu'il s'agit du prédicat. Cette procédure, couteuse en temps et en énergie cognitive, est tributaire d'un ensemble de sous-procédures, dont l'identification du verbe, et semble donc engendrer des difficultés chez Pierre.

Le sentiment d'efficacité personnelle en grammaire des élèves du groupe 2 est généralement plus bas que celui des participants du premier groupe. Isaac souligne dans 
l'entrevue qu'il ne se trouve pas compétent en grammaire parce ce n'est pas une discipline qui l'intéresse et qu'il préfère en fait les mathématiques. Quand on lui pose la question relativement à ce qu'il trouve facile en grammaire, il répond en mobilisant un argument lié à la fréquence d'occurrences de certaines formes grammaticales.

Expérimentateur : Qu'est-ce qui est facile pour toi en grammaire ?

Isaac: Conjuguer au présent.

Expérimentateur: Okay, pourquoi tu trouves ça facile ?

Isaac: Car euh, on utilise le présent beaucoup de fois dans la vie. Comme $j e, t u, i l$, elle, des choses comme ça, on utilise ça beaucoup dans la vie.

De tout notre échantillon, Isaac est le seul qui recourt à ce type de critères pour expliquer ce qui, pour lui, est facile en grammaire.

Celle dont le score est le plus élevé dans le groupe 2, Maya, souligne qu'elle éprouve une certaine facilité à repérer le sujet et le complément de phrase.

Expérimentateur : Pourquoi c'est facile trouver le complément de phrase?

Maya : Euh, car euh, qu'est-ce qu'on doit vraiment faire est, trouver un groupe de mots et voir comme, si on peut le bouger à une autre place... I'm not sure how to say it in French.

Expérimentateur : Not a problem. Why is it easy to find this complement?

Maya: It's easier because, when I move it, it helps me understand the sentence better sometimes.

Nous relevons plusieurs points intéressants dans cet extrait. D'abord, les manipulations syntaxiques semblent faciliter l'apprentissage de la grammaire pour Maya et, plus précisément, l'analyse de phrases. Il appert aussi que, grâce à ces procédures métalinguistiques, elle peut, avec plus d'aisance, faire sens des énoncés qu'elle analyse. On retrouve donc encore une fois les bienfaits d'un enseignement grammatical sur l'apprentissage de la lecture. Concernant ses difficultés, elle précise qu'elle ne connait pas toujours le genre des noms qu'elle utilise en production.

Dans le groupe 3, Ali se démarque cette fois-ci, non pas parce que, comme pour les autres indicateurs du questionnaire de la dynamique motivationnelle en grammaire, il obtient l'un des scores les plus bas, mais bien parce que, en ce qui a trait au sentiment d'efficacité personnelle, il obtient le score maximal. Concernant ses facilités, il juge l'analyse de phrases comme étant facile.

Expérimentateur : Qu'est-ce qui est facile pour toi en grammaire.

Ali : L'analyse de phrases.

Expérimentateur: Okay, pourquoi ?

Ali : Parce que c'est juste facile, parce que la seule chose que tu dois faire c'est comme, comprendre la phrase, tu dois juste savoir le sujet. Comme, quand tu sais le sujet, tu sais de quoi la phrase va parler à propos de. Et tu vas savoir où est le verbe parce que le verbe est toujours avant ou derrière le sujet. 
Dans ce passage, Ali nous montre qu'il connait les outils qui permettent l'analyse des phrases françaises et que leur mise en application lui facilite l'effort de réflexion grammaticale $^{3}$. Pour lui, ce sont les efforts de mémorisation qu'il doit déployer qui lui engendrent des difficultés.

\section{Discussion}

Au regard des données présentées dans cet article, on peut dire que les liens entre le développement de la compétence qui permet l'accord verbal à l'écrit chez nos participants et leur motivation en grammaire sont relativement étroits. En effet, les scores généraux obtenus au questionnaire en motivation montrent que les élèves du groupe 1 sont plus motivés dans leur apprentissage grammatical, alors que les élèves du groupe 2 le sont moins. Les élèves du groupe 3, pour leur part, présentent des résultats mitigés : Alicia semble relativement motivée, tandis qu'Ali obtient un score de 0/24. Conformément à plusieurs travaux sur la motivation et l'écriture (Saddler, 2012; Troia, Shankland et Wolbers, 2012), lesquels discutent les effets positifs d'une motivation élevée en écriture sur la qualité des productions écrites, nous posons l'hypothèse qu'un tel lien existe aussi entre la motivation en grammaire et le développement de la compétence à écrire le verbe.

Nos données nous permettent de surcroit d'offrir un éclairage relatif aux indicateurs qui, dans notre étude, permettent de rendre compte de la dynamique motivationnelle de nos élèves en grammaire : l'intérêt en grammaire, la perception de l'utilité de la grammaire et le sentiment d'efficacité personnelle en grammaire. Pour ce qui est de l'intérêt, les résultats au questionnaire varient d'un apprenant à l'autre, mais les propos qu'ils tiennent pendant l'entrevue permettent de faire émerger certaines tendances. En effet, les élèves du groupe 1 , plus compétents en grammaire dès le début de l'étude, savent cibler les concepts qui suscitent chez eux un intérêt et ceux qui, à contrario, constituent une source d'ennui. Ils font usage de termes métalangagiers de manière plus fréquente pour expliquer leurs points de vue et semblent prendre plaisir à adopter une posture réflexive à l'endroit des unités constitutives de la langue. Enfin, l'ensemble des élèves de notre échantillon déclare que les efforts de mémorisation qu'ils doivent parfois déployer lors de l'apprentissage de certains concepts ne contribuent guère à leur intérêt vis-à-vis de la grammaire.

De manière générale, les scores obtenus quant aux perceptions à l'égard de l'utilité de la grammaire sont plus élevés que ceux concernant leur intérêt. Cet état de fait corrobore les résultats de Jean et Simard (2011) qui, en langue seconde, ont remarqué que l'apprentissage de la grammaire renvoyait pour les élèves à un mal nécessaire, qu'elle serait utile, mais ennuyeuse. Les élèves du groupe 1, dont la compétence est la plus élevée au moment où nous avons décrit la dynamique motivationnelle des participants en grammaire, déclarent que leur apprentissage de la grammaire leur permet de rehausser la qualité de leurs productions écrites et, globalement, ils sont capables de cibler, comme nous l'avons remarqué dans les extraits de verbatim abordant leur intérêt en grammaire, les concepts grammaticaux les plus utiles. Les élèves du groupe 2, plus faibles, font aussi mention de l'apport de la grammaire pour la qualité de leurs productions, mais ne se focalisent pas sur les faits de langue. Enfin, les élèves du groupe 3, qui développeront quelques mois après

\footnotetext{
${ }^{3}$ Fait intéressant, au moment où Ali a pris part à cet entretien, il n'utilise pratiquement pas les manipulations syntaxiques quand il accorde ses verbes. Par contre, Ali est celui qui, quelques mois plus tard, en février 2016, fait un usage presque systématique de ces manipulations lors des tâches portant sur le verbe et son accord.
} 
la description de leur dynamique motivationnelle en grammaire une compétence à accorder les verbes tout à fait opérationnelle, en mentionnent des bénéfices différents de leurs pairs ; Ali aborde explicitement l'association des phonèmes aux graphèmes, alors qu'Alicia parle de l'apport de la grammaire dans la construction de sens.

Enfin, le sentiment d'efficacité personnelle en grammaire de tous les élèves du groupe 1 est positif, alors que, dans le groupe 2, il se situe en deçà de 0/24 pour Kate et Isaac. Ce résultat rappelle celui de Boyer (2012), qui a montré que ce sentiment pouvait être influent sur l'apprentissage orthographique. Dans le groupe 3, le score d'Ali, en ce qui a trait à cet indicateur, mérite aussi d'être discuté. En effet, pour tous les autres indicateurs de la motivation, ce participant obtient un score négatif, alors qu'il atteint le score maximal pour ce qui est de son sentiment d'efficacité personnelle en grammaire. Ali, qui peaufinera sa compétence liée à l'accord du verbe de manière remarquable à la suite de notre description de sa dynamique motivationnelle en grammaire, nous rappelle donc les résultats de chercheurs (Bandura, 1997; Bouffard et Narciss, 2011) soutenant que l'illusion de compétence et d'incompétence devient fréquemment réalité. Par ailleurs, les propos qu'ils tiennent lors de l'entrevue nous indiquent que plusieurs élèves - Sabrina, Emma, Ali et Maya - trouvent l'analyse phrastique facile, notamment parce qu'ils peuvent recourir à des outils d'analyse pour mener à bien un tel travail grammatical. Enfin, à l'exception de Pierre, tous les élèves peinent à s'investir dans un effort de mémorisation quand ils font de la grammaire à l'école.

\section{Conclusion}

Nous ne saurions, en raison du devis qualitatif qui a été retenu, suggérer la représentativité des données qui sont ici présentées. Cela étant, nos résultats nous encouragent à poursuivre la réflexion sur la motivation et son rôle dans l'apprentissage grammatical. Ainsi, à la lumière de nos résultats, il nous semble important que les enseignants mettent en place des pratiques qui rendent les élèves cognitivement actifs lors de leur apprentissage et qui traitent la langue non pas comme un amas de composantes qui n'entretiennent pas de liens entre elles, mais bien comme un tout unifié, cohérent et, ipso facto, accessible. Les apprenants pourront dès lors comprendre, de manière féconde, les rouages du système par l'intermédiaire duquel se réalisent les discours qu'ils produisent et devraient donc déployer des efforts de mémorisation moins considérables pour le mettre en application.

\section{Références}

Bandura, A. (1997). Self-efficacy. The exercice of control. New York, NY : Freeman.

Baribeau, C. et Royer, C. (2012). L'entretien individuel en recherche qualitative : usages et modes de présentation dans la Revue des sciences de l'éducation. Revue des sciences de l'éducation, 38(1), 23-45.

Bélanger, M., Minor-Corriveau, M. et Bélanger, R. (2015). A comparison of the frequency and the types of French spelling errors produced by students located in different demolinguistic settings. The International Journal of Assessment and Evaluation, 22(4), 17-27.

Blain, S. (2003). L'enseignement de l'écriture en milieu minoritaire canadien : problématique particulière et complémentarité des cadres théoriques en L1 et L2. 
Dans J.-M. Defays, J.-L. Delcominette, J.-L. Dumortier et V. Louis (dir.), Langue et communication en classe de français. Convergences didactiques en langue maternelle, langue seconde et langue étrangère (p. 185-200). Cortil-Wodon, Belgique : Éditions Modulaires Européennes.

Boscolo, P. et Hidi, S. (2007). The multiple meanings of motivation to write. Dans S. Hidi et P. Boscolo (dir.), Writing and motivation (p. 1-16). Oxford, Angleterre : Elsevier.

Bouffard, T. et Narciss, S. (2011) Benefits and risks of positive biases in self-evaluation of academic competence: Introduction. Journal of Educational Research, 50(4), 205-208.

Boyer, P. (2012). La compétence morphographique d'élèves de première secondaire. $L$ 'effet du biais d'évaluation de son efficacité personnelle sur la performance (thèse de doctorat, Université du Québec à Montréal, Canada). Récupéré le 5 février 2017 d'Archipel, le dépôt numérique de 1'Université du Québec à Montréal : http://www.archipel.uqam.ca/5352/1/D2405.pdf

Bruning, R., Dempsey, M., Kauffman, D. F., McKim, C. et Zumbrunn, S. (2013). Examining dimensions of self-efficacy for writing. Journal of Educational Psychology, 105(1), 25-38.

Cavanagh, M. et Blain, S. (2009). Relever quatre défis de l'enseignement de l'écrit en milieu francophone minoritaire. Cahiers franco-canadiens de l'Ouest, 21(1-2), 151-178.

Chartrand, S.-G., Aubin, D., Blain, R. et Simard, C. (2011). Grammaire pédagogique du français d'aujourd'hui (2 éd.). Montréal, Canada : Chenelière Éducation.

Cogis, D. (2013). Du prescrit au réel en CM2 : l'accord sujet-verbe dans le corpus Grenouille. Dans C. Gunnarsson-Largy et E. Auriac-Slusarczyk (dir.), Écriture et réécritures chez les élèves. Un seul corpus, divers genres discursifs et méthodologies d'analyse (p. 61-84). Louvain-la-Neuve, Belgique : AcademiaL'Harmattan.

David, J. et Renvoisé, C. (2010). La morphologie verbale : repérer les complexités et les régularités. Synergies France, 6, 61-75.

Geoffre, T. et Brissaud, C. (2012). L'accord sujet-verbe : acquis en fin d'école primaire, vraiment? Récupéré le 28 avril 2017 du site de SHS : http://www.shsconferences.org/articles/shsconf/pdf/2012/01/shsconf_cmlf12_000196.pdf

Groupe DIEPE (1995). Savoir écrire au secondaire. Étude comparative auprès de quatre populations francophones d'Europe et d'Amérique. Bruxelles, Belgique : De Boeck.

Harter, S. (1999). The development of self-representations: A developmental perspective. New York, NY : Guilford Press.

Hayes, J. R. et Flower, L. (1980). Identifying the organization of writing processes. Dans L. W. Gregg et E. R. Steinberg (dir.), Cognitive processes in writing. Hillsdale, MI: Lawrence Erlbaum.

Hidi, S., Berndorff, D. et Ainley, M. (2002). Children's argument writing, interest, and self-efficacy: An intervention study. Learning and Instruction, 12(4), 429-446.

Jean, G. et Simard, D. (2011). Grammar teaching and learning in L2: necessary, but boring? Foreign Language Annals, 44(3), 467-494. 
Kaufman, A. S. et Kaufman, N. L. (1995). L'examen psychologique de l'enfant, $K-A B C$, pratiques et fondements théoriques. Aubenais, France : La pensée sauvage.

Lefrançois, P., Montésinos-Gelet, I. et Anctil, D. (2018). Enseigner la phrase par la littérature jeunesse. Fondements et activités pour soutenir la compétence à écrire au primaire. Montréal, Canada : Chenelière Éducation.

Lord, M.-A. (2012). L'enseignement grammatical au secondaire québécois : pratiques et représentations d'enseignants de français (thèse de doctorat, Université Laval, Canada). Récupéré le 5 février 2017 du dépôt numérique de l’Université Laval : https://corpus.ulaval.ca/jspui/handle/20.500.11794/23457

Ministère de l'Éducation de l'Ontario (2006). Le curriculum de l'Ontario de la première à la huitième année. Français. Récupéré le 5 février 2017 du site du ministère : http://www.edu.gov.on.ca/fre/curriculum/elementary/language18currb.pdf

Murray, B. (2016). Le rapport à l'écrit en français et en anglais d'étudiants francophones universitaires issus d'un milieu francophone minoritaire (mémoire de maitrise, Université d'Ottawa, Canada). Récupéré d'uO Recherche, le dépôt numérique de l'Université d'Ottawa : https://ruor.uottawa.ca/bitstream/10393/35004/1/Murray\%20_Brigitte_2016 these . $\mathrm{pdf}$

Nelson, N. (2012). No final word: Cognition and context in writing research. Dans C. Gelati, B. Arté, et L. Mason (dir.), Essays in writing research: In honor of Piero Boscolo (p. 13-27). Padoue, Italie : Università Degli Studi di Padova.

Ontario 400 (2016). Statistiques. Récupéré le 5 février 2017 du site d'Ontario 400 : http://ontario400.ca/statistiques/

Pajares, F., Britner, S. L. et Valiante, G. (2000). Relation between achievement goals and self-beliefs of middle school students in writing and science. Contemporary Educational Psychology, 25(4), 406-422.

Saddler, B. (2012). Motivating writers: Theory and interventions. Reading and Writing Quarterly, 28(1), 1-4.

Thibeault, J. (2016). Arrimer les cadres théoriques en langues première et seconde pour décrire les connaissances de l'écrit d'élèves en milieu francophone minoritaire : l'exemple de l'accord du verbe en nombre chez des scripteurs de l'élémentaire en Ontario français. Formation et profession, 24(2), 68-71.

Thibeault, J., Fleuret, C. et Lefrançois, P. (2018). Développement de la compétence linguistique permettant l'accord du verbe en nombre à l'écrit chez des élèves de l'élémentaire en contexte minoritaire. Revue canadienne de linguistique appliquée, 21(2), 19-45.

Thibeault, J. et Lefrançois, P. (2018). Exploration de l'évolution des commentaires métagraphiques relatifs à l'accord du verbe en nombre chez des élèves de la fin de l'élémentaire scolarisés dans le Sud-Ouest ontarien. Revue canadienne des langues vivantes, 74(4), 575-602.

Troia, G. A., Shankland, R. K. et Wolbers, K. A. (2012). Motivation research in writing: Theoritical and empirical considerations. Reading and Writing Quarterly, 28(5), 5-28.

Van der Maren, J.-M. (2004). Méthodes de recherches pour l'éducation (2e éd.). Bruxelles, Belgique : De Boeck.

Villalón, R., Mateos, M. et Cuevas, I. (2015). High school boys' and girls' writing 
conceptions and writing self-efficacy beliefs: What is their role in writing performance? Educational Psychology, 35(6), 653-674.

Wigfield, A. et Eccles, J. (1992). The development of achievement task values: A theoretical analysis. Developmental Review, 12(3), 265-310.

Zimmerman, B. J. et Kitsantas, A. (2007). A writer's discipline: The development of selfregulatory skill. Dans S. Hidi et P. Boscolo (dir.), Writing and motivation (p. 51-

69). Oxford, Angleterre : Elsevier.

Biographie des auteurs

Joël Thibeault est professeur adjoint à la Faculté d'éducation de l'Université de Regina. Ses travaux portent sur l'enseignement de l'écriture et de la grammaire.

Carole Fleuret est professeure agrégée à la Faculté d'éducation de 1'Université d'Ottawa. Ses recherches ont trait à l'enseignement et à l'apprentissage de l'écrit en contextes plurilingues. 